\title{
Detection of Glutamate and Acetylcholine with Organic Electrochemical Transistors Based on Conducting Polymer/Platinum Nanoparticle Composites
}

Loig Kergoat, Benoit Piro, Daniel Simon, Vincent Minh-Chau Pham; Noel and Magnus

Berggren

\section{Linköping University Post Print}

\section{Tweet}

N.B.: When citing this work, cite the original article.

Original Publication:

Loig Kergoat, Benoit Piro, Daniel Simon, Vincent Minh-Chau Pham; Noel and Magnus Berggren, Detection of Glutamate and Acetylcholine with Organic Electrochemical Transistors Based on Conducting Polymer/Platinum Nanoparticle Composites, 2014, Advanced Materials, (26), 32, 5658-5664.

http://dx.doi.org/10.1002/adma.201401608

Copyright: Wiley-VCH Verlag

http://www.wiley-vch.de/publish/en/

Postprint available at: Linköping University Electronic Press http://urn.kb.se/resolve?urn=urn:nbn:se:liu:diva-110967 


\section{WILEY-VCH}

DOI: $10.1002 /(($ please add manuscript number $))$

Article type: Communication

\section{Detection of Glutamate and Acetylcholine with Organic Electrochemical Transistors Based on Conducting Polymer/Platinum Nanoparticles Composites}

Loïg Kergoat, Benô̂t Piro, Daniel T. Simon, Minh-Chau Pham, Vincent Noël*, Magnus Berggren*

Dr. L. Kergoat, Dr. D. T. Simon, Prof. M. Berggren

Linköping University, ITN, Laboratory of Organic Electronics, SE-601 74, Sweden

E-mail: magnus.berggren@liu.se

Pr. B. Piro, Pr. M. Pham, Dr. V. Noël

Univ. Paris Diderot, Sorbonne Paris Cité, ITODYS, UMR 7086 CNRS, 15 rue J-A de Baï, 75205 Paris Cedex 13, France

E-mail: vincent.noel@univ-paris-diderot.fr

Keywords: (bioelectronics, neurotransmitters, PEDOT:PSS, platinum nanoparticles, electrochemical transistors)

According to the World Health Organization, ${ }^{[1]}$ approximately 1 in every 100 of the world's inhabitants suffers from some form of neurological disorder such as epilepsy, Parkinson's disease, or Alzheimer's disease. These diseases arise from malfunctioning in the neurochemical signalling. Today's methods of treatment of those diseases focus primarily on pharmaceutical and electroceutical neuromodulation techniques to supress the effects of the disease or to restore healthy signalling. Both pharmaceutical (e.g., levodopa therapy) and electroceutical (e.g., deep brain stimulation [DBS]) therapies are associated with varying degrees of success. However, all therapies rely on the calibration of a delivery dose primarily dictated via external monitoring of the patient's symptoms and physiological state. A technology is lacking that enables the administration of chemical and electrical signals at high precision, in terms of spatiotemporal resolution, pharmaceutical specificity and dosage that takes the patient's specific physiological condition into an account.

Signalling in the nervous system is based on gradients and transport of neurotransmitters (intercellular) and ions (intracellular). Abnormal neurotransmitter concentrations can reveal the state of a disease and can also be an early indicator of for instance a seizure. For example, 


\section{WILEY-VCH}

elevated levels of glutamate, the primary excitatory neurotransmitter in the central nervous system (CNS), may indicate disorders such as epilepsy, amyotrophic lateral sclerosis or Parkinson's disease ${ }^{[2]}$ Similarly, abnormal levels of acetylcholine, a key signalling entity in learning and memory of the CNS and in the triggering and control of muscle activity of the peripheral nervous system, may indicate disorders such as Alzheimer's disease ${ }^{[3]}$ or dementia. ${ }^{[4]}$ Significant effort has therefore been devoted in recent years for the development of highly sensitive and specific techniques to detect local neurotransmitter concentrations. Neurotransmitters, such as glutamate and acetylcholine, can be detected using microdialysis techniques that can be combined with high performance liquid chromatography (HPLC). ${ }^{[5,6]}$ The main drawback of such analytical method is that they require sample collection for $e x$ vivo analysis, severely limiting sensing speed and convenience. Electrochemical detection of $\mathrm{H}_{2} \mathrm{O}_{2}$, resulting from the enzymatic degradation of the neurotransmitter substrate, overcomes this issue by locally transducing changes in chemical concentration into fluxes of electrons. Electronic signal can easily be recorded and monitored in real time. However, $\mathrm{H}_{2} \mathrm{O}_{2}$ charge transfer kinetics is slow on most of conductive substrates. Platinum is known for its catalytic activity in the oxidation of $\mathrm{H}_{2} \mathrm{O}_{2} \cdot{ }^{[7]}$ Indeed, platinum micro-electrodes have recently been used to detect both glutamate and acetylcholine in vivo. ${ }^{[8]}$ This work has paved the way for efficient in-situ monitoring of neurotransmitters, in part thanks to the excellent electrocatalytic activity of $\mathrm{Pt}$ toward $\mathrm{H}_{2} \mathrm{O}_{2}$ oxidation. Previous studies show that the improvement of the electrocatalytic activity of Pt in terms of overpotential and sensitivity can be achieved by the use of surface-supported nanosized Pt owing to its larger surface area. ${ }^{[9-10]}$ Several strategies were used to take advantages of such Pt nanostructures, such as Pt black electrodeposition, Pt nanoparticles (Pt NPs) inclusion in C films and Pt NPs embedded in organic corona Langmuir-Blodgett films. The latter allows for fine-tuning of the thickness and also the physico-chemical properties of the organic corona (charge, hydrophobic/hydrophilic balance,...) and hence the ability to achieve Pt NPs dispersion within various matrices. Recent 


\section{WILEY-VCH}

publication ${ }^{[11]}$ shows that the electrocatalytic properties toward $\mathrm{H}_{2} \mathrm{O}_{2}$ oxidation are maintained despite the organic corona and efficient even at very low Pt NPs surface content. Effort has been devoted to develop 3D-structured materials by incorporating the Pt NPs into electronically conducting polymers, such as poly(ethylene dioxythiophene) doped with poly(styrene sulfonate) (PEDOT:PSS). ${ }^{[12]}$ PEDOT:PSS is a very interesting support electronic hydrogel matrix due to its high degree of porosity (substrate accessibility) and its high electrical conductivity (electronic wiring of Pt NPs). However, most of these studies target energy conversion and energy storage applications.

Nevertheless, several conducting polymers, such as PEDOT:PSS, are very interesting material systems for biological and medical applications due to their ability to transport both ionic and electronic charges. Their porous structure allows ions to penetrate into their bulk, allowing ionic exchange between the organic material and an aqueous surrounding medium, which combined to their biocompatibility, makes them perfect candidates for many biological applications. This ionic exchange is controlled by the electric current applied to the device and generally causes a change of the redox states, leading to a change in the electronic conductivity. The possibility to electronically control the ionic flux inside the polymer has been used for active drug ${ }^{[13]}$ and neurotransmitter delivery devices. ${ }^{[14]}$ Organic electrochemical transistors (OECTs) take advantage of the change of electrical conductivity, resulting from the ionic exchange between the conducting polymer system and the electrolyte. The first OECT was developed using polypyrrole by Whrighton et al. in the mid-eighties. ${ }^{[15]}$ In a similar way to organic field effect transistors (OFET), OECTs are threeterminal devices. The channel between the source and drain consists of an electrochemically active conducting polymer and the third electrode, serving as the gate, is separated from the channel by an electrolyte. The working principle of OECTs is based on the doping/dedoping of the channel upon gate polarization. Nowadays, PEDOT:PSS is the benchmark polymer used as the channel material in OECTs. In it pristine state (oxidised), PEDOT:PSS is 


\section{WILEY-VCH}

conducting. When the gate is positively biased, reduction of the conducting polymer occurs at the negatively polarised channel reducing the electrical conductivity of the polymer. To compensate for the gain of electrons, cations from the electrolyte penetrate in the channel, . The popularity of PEDOT:PSS comes from the fact that it can be solution-processed and therefore be manufactured using low cost procedures, such as printing techniques, ${ }^{[19]}$ on a wide variety of substrates (plastic, ${ }^{[16,17]}$ paper, ${ }^{[18]}$ woven fabric, ${ }^{[19]} \ldots$ ), is commercially available, has a relatively high conductivity and is proven to be biocompatible. ${ }^{[20]}$ Recently, OECTs have been used to create cell-density gradients, ${ }^{[21]}$ to measure barrier tissue integrity ${ }^{[22]}$ and to monitor action potentials in rat brains, ${ }^{[23]}$ but also much efforts have been dedicated to utilize PEDOT:PSS in OECTs for biosensor applications. OECTs have been used for the detection of DNA, ${ }^{[24]}$ dopamine ${ }^{[25]}$ and bacteria. ${ }^{[26]}$ However, most of the studies cope with the enzymatic detection of glucose with glucose oxidase using bulk platinum as gate material $^{[27,28]}$. Sensitivity and limit of detection were further improved using nanomaterials (carbon nanotubes and Pt NPs) modified Pt gate electrode. ${ }^{[29]}$

In this work, we focus on the use of a PEDOT:PSS/Pt NPs OECTs (Figure 1 a) for the enzymatic detection of two of the main neurotransmitters in the human body, glutamate and acetylcholine. One key point in the development of an enzyme biosensor is the stable attachment of the enzyme onto the surface of - or embedded within - the sensing material. This process is governed by various interactions between the enzyme and the material and strongly affects the performance of the biosensor in terms of sensitivity, stability, response time, and reproducibility. In this context, appropriate selection of the electrode material and the immobilization chemistry are essential for a reliable biosensor, as discussed in recent reviews ${ }^{[30]}$ and articles ${ }^{[31,32]}$. In this initial study, we chose to localize enzymes at the PEDOT:PSS/solution interface and to use a robust and easy-to-implement immobilization strategy based on enzyme crosslinkers. 


\section{WILEY-VCH}
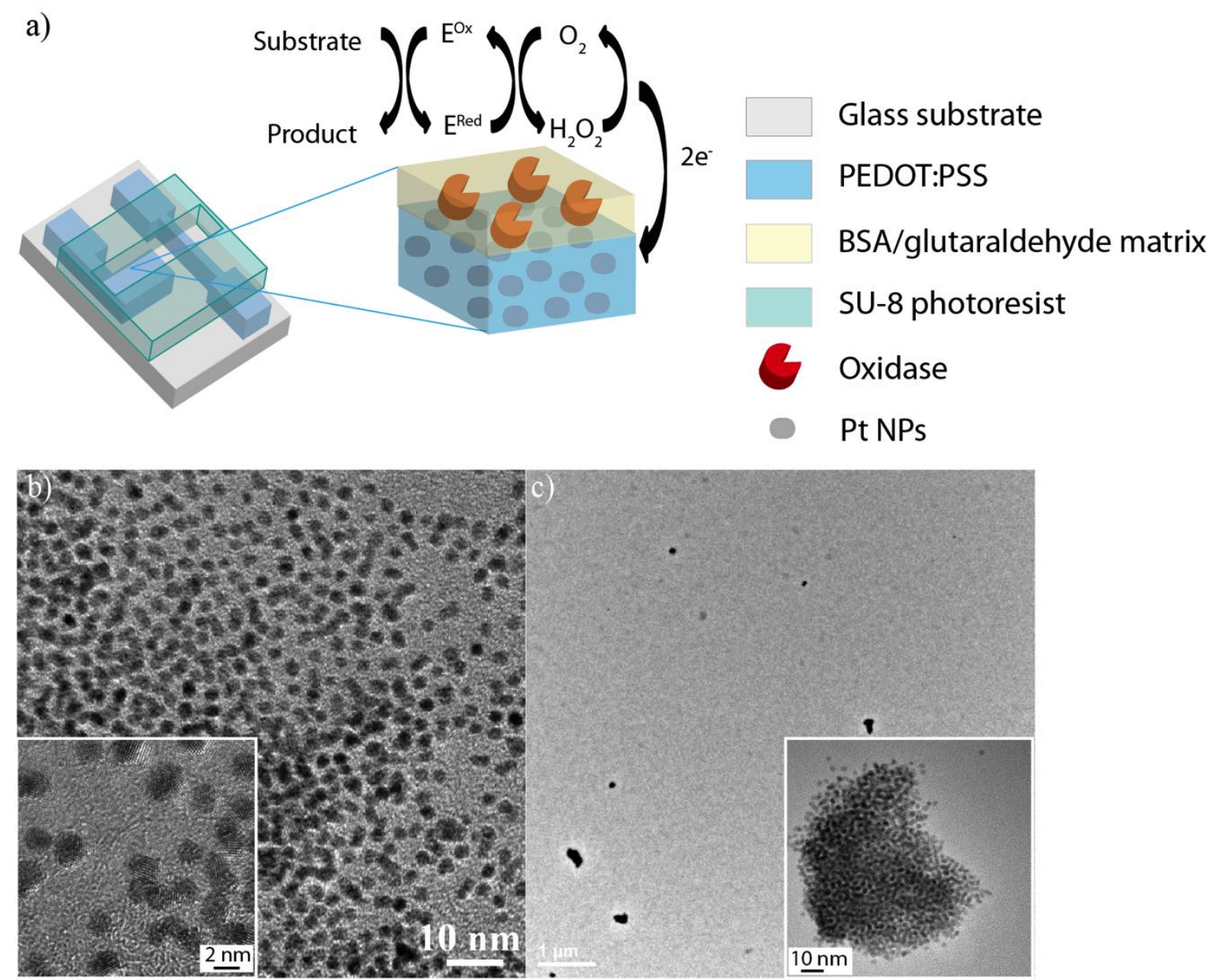

Figure 1. a) Schematic diagram of the OECT neurotransmitter sensor and the enzymatic

sensing mechanism . TEM pictures obtained at different magnifications from b) the Pt NPs dispersed in DMSO and c) of the PEDOT:PSS/Pt NPs composite film.

TEM measurements were performed on freshly synthesized Pt NPs dispersed in DMSO (Figure 1b). The diameter of the nanoparticles is about $2.3 \mathrm{~nm}$ and the $10 \mathrm{~nm}$-scale image shows a relatively mono-disperse ensemble. The $2 \mathrm{~nm}$-scale image (inset Figure 1b) reveals a diffraction pattern indicating that the nanoparticles are crystalline. TEM pictures of the PEDOT:PSS/PtNPs composite film (Figure 1c) indicate that the nanoparticles tend to aggregate and form clusters when mixed with the PEDOT:PSS matrix. The size of these clusters is typically about $200 \mathrm{~nm}$ with several micrometers separating the clusters. The presence of these clusters could be explained by the fact that the PEDOT:PSS solution is composed of approximately $99 \%$ water and that the NPs do not disperse well in water. 


\section{WILEY-VCH}

Electrochemical detection involving the use of an oxydoreductase typically occurs according to the mechanism depicted in Figure 1a. The substrate is oxidised by the enzyme $\left(\mathrm{E}^{\mathrm{ox}}\right)$ into a product. The reduced enzyme $\left(\mathrm{E}^{\mathrm{red}}\right)$ is then reoxidised by oxygen, leading to the formation of $\mathrm{H}_{2} \mathrm{O}_{2}$. Finally $\mathrm{H}_{2} \mathrm{O}_{2}$ is reoxidised into oxygen at the electrode. Therefore, we first studied the response of our composite film to $\mathrm{H}_{2} \mathrm{O}_{2}$.

Voltammograms (Figure 2a) were recorded for a pristine film of PEDOT:PSS and a composite film of PEDOT:PSS/Pt NPs in phosphate buffered saline solution (PBS) in the presence of $1 \mathrm{mM} \mathrm{H}_{2} \mathrm{O}_{2}$. Both curves show a relatively high capacitive component due to that charges can be compensated through out the entire electrode bulk (super-capacitance). For the composite film of PEDOT:PSS/Pt NPs, an irreversible reduction wave starts at ca. $0 \mathrm{~V}$ which can be attributed to the electrocatalytic activity of the Pt NPs towards $\mathrm{H}_{2} \mathrm{O}_{2}$. Symmetrically, in the positive potential domain, the catalytic two-electron $\mathrm{H}_{2} \mathrm{O}_{2}$ oxidation on Pt occurs from ca. $0.25 \mathrm{~V}$ vs $\mathrm{Ag} / \mathrm{AgCl}$ as already described in the literature for ensemble of Pt NPs. ${ }^{[1]}$ This behaviour indicates that Pt NPs are accessible and electronically active and also connected to the ITO underlying electrode via the PEDOT:PSS matrix.

Chronoamperograms were recorded to monitor the response of the electrodes upon addition of various concentrations of $\mathrm{H}_{2} \mathrm{O}_{2}$. A potential of $+0.4 \mathrm{~V}$ vs $\mathrm{Ag} / \mathrm{AgCl}$ was applied to the electrode. After stabilization of the current, the concentration of $\mathrm{H}_{2} \mathrm{O}_{2}$ in the electrolyte was gradually increased. Chronoamperograms are shown for the pristine PEDOT:PSS on ITO (Figure 2b) and for the composite films PEDOT:PSS/PT NP on ITO (inset Figure 2b). The addition of $\mathrm{H}_{2} \mathrm{O}_{2}$ does not induce any modification in the response of the pristine film up to a concentration of $145 \mu \mathrm{M} \mathrm{H}_{2} \mathrm{O}_{2}$. For the composite film, a slight increase in the current density is observed from $1.6 \mu \mathrm{M} \mathrm{H}_{2} \mathrm{O}_{2}$ but for a concentration of $6.2 \mu \mathrm{M}$ a clear sensor response is observed. 
a)

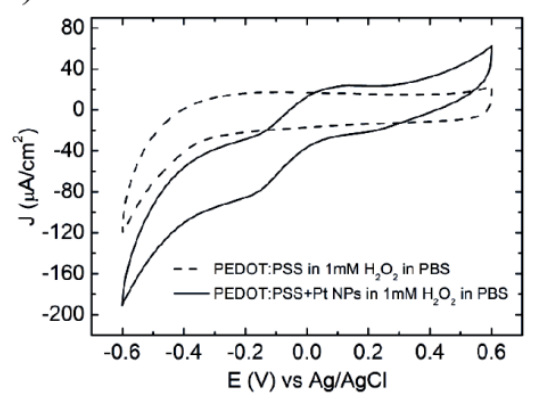

c)

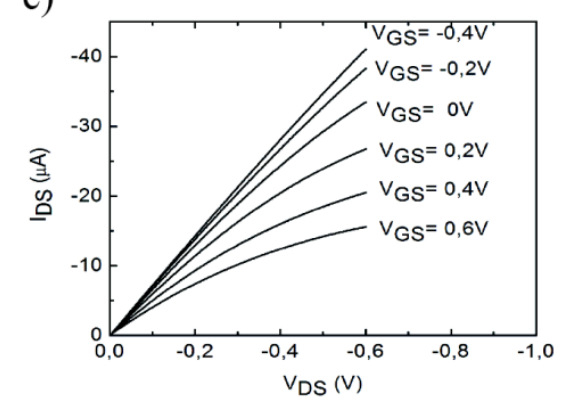

b)

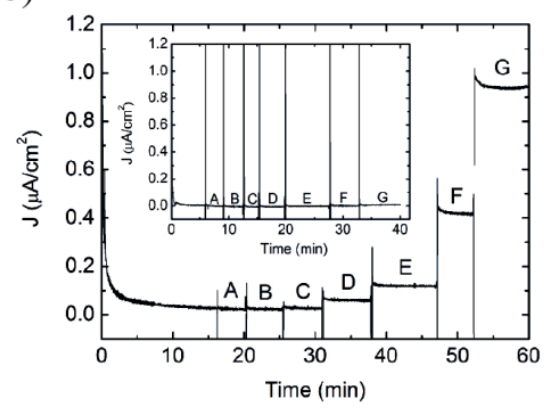

d)

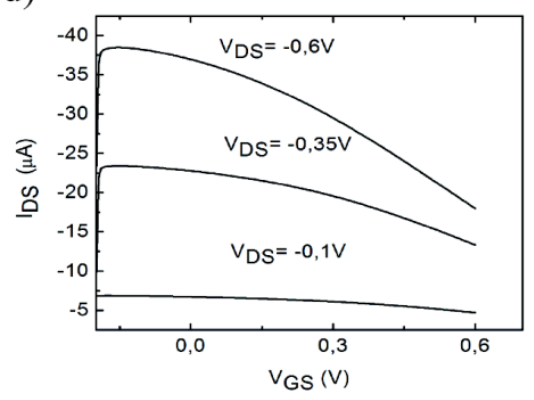

Figure 2. a) Cyclic voltammograms of the pristine PEDOT:PSS film (dashed line) and the composite PEDOT:PSS/Pt NPs film (full line) on ITO obtained at $50 \mathrm{mV} . \mathrm{s}^{-1}$ in $1 \mathrm{mM} \mathrm{H}_{2} \mathrm{O}_{2}$ in PBS. b) Chronoamperograms obtained at $+0.4 \mathrm{~V}$ for the PEDOT:PSS/Pt NPs composite film and (Inset) for the pristine PEDOT:PSS film for increasing concentration of $\mathrm{H}_{2} \mathrm{O}_{2}$ in PBS $(\mathrm{A}=0.1 \mu \mathrm{M}, \mathrm{B}=0.6 \mu \mathrm{M}, \mathrm{C}=1.6 \mu \mathrm{M}, \mathrm{D}=6.2 \mu \mathrm{M}, \mathrm{E}=14 \mu \mathrm{M}, \mathrm{F}=57 \mu \mathrm{M}, \mathrm{G}=145 \mu \mathrm{M}) . c)$ Output and d) transfer characteristics of a PEDOT:PSS/Pt NPs OECT using PBS as electrolyte.

OECTs were patterned using photolithography. All three electrodes and the channel were composed of the PEDOT:PSS/Pt NPs composite film (Figure 1 a) and patterned simultaneously. The channel length and width were $2500 \mu \mathrm{m}$ and $500 \mu \mathrm{m}$, respectively, giving a channel surface area of $0.0125 \mathrm{~cm}^{2}$. The gate area was $0.075 \mathrm{~cm}^{2}$. An SU-8 layer was patterned to define the active area in contact with the electrolyte and carbon paste was used on the contacts to improve the connection between the PEDOT:PSS and the measurement probes. To reduce the number of photolithography steps, one could easily replace the SU-8 layer by a PDMS well, or other gasket material with a pre-cut shape to define the electrolyte area.

However, using SU-8 provides a high degree of precision and a better reproducibility between different devices. The output characteristics of the OECT (Figure 2c) were recorded by 


\section{WILEY-VCH}

sweeping the drain voltage between $0 \mathrm{~V}$ and $-0.6 \mathrm{~V}$. The gate voltage was changed from $-0.4 \mathrm{~V}$ to $0.6 \mathrm{~V}$ with a $0.2 \mathrm{~V}$ step. Applying a positive gate voltage decreases the drain current, a behaviour that is consistent with the classical operation mode of PEDOT:PSS-based OECTs. ${ }^{[28]}$ The corresponding transfer curve is presented Figure $2 \mathbf{d}$.

Typically, enzymatic detection with an OECT involving an oxydoreductase is done by the anodic detection of hydrogen peroxide, generated by the reoxidation of the enzyme, at a platinum gate electrode. However, cyclic voltammetry (Figure 2a) showed oxidation and reduction peaks at low potential for our composite PEDOT:PSS/Pt NPs film. As described above, OECTs are operated by applying a negative voltage between source and drain and a positive voltage to the gate. Therefore, the reduction of hydrogen peroxide at the negatively biased channel is also likely to occur during OECT operation (Figure 3a). 
a)

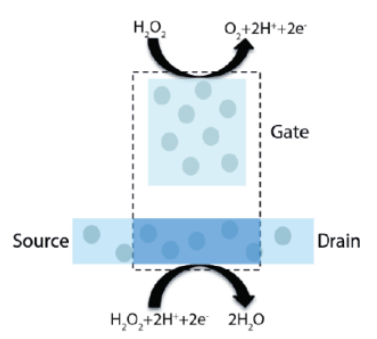

c)
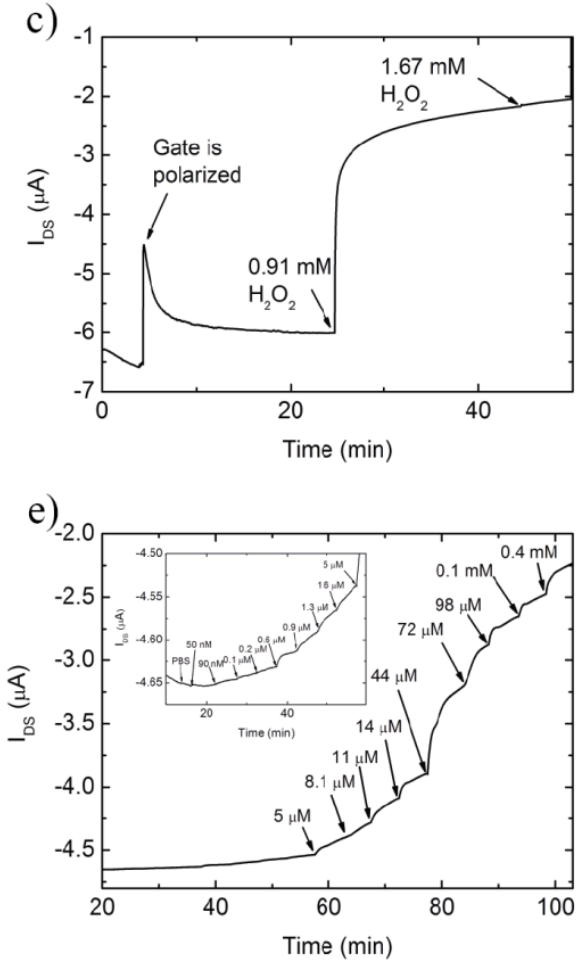

b)

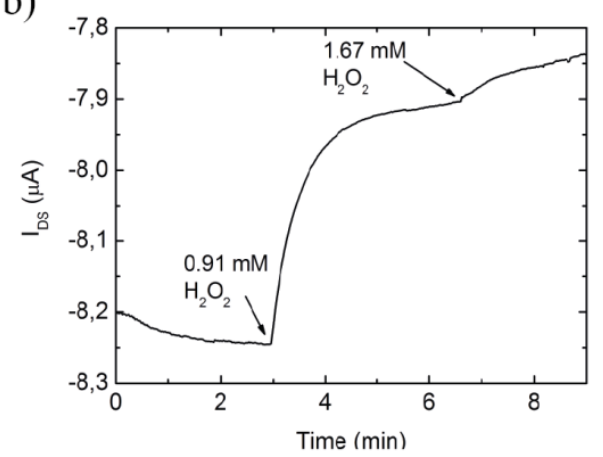

d)

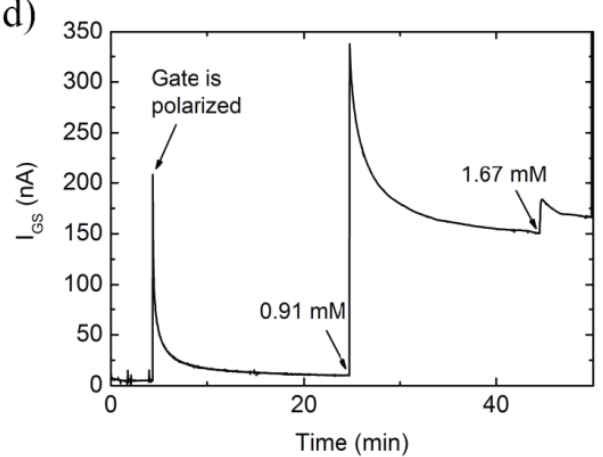

f)

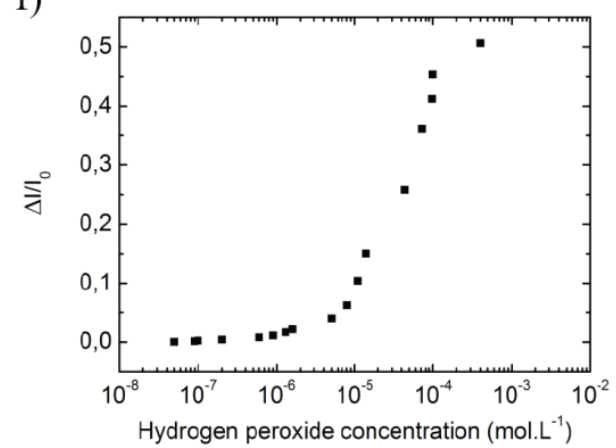

Figure 3. a) Schematic representation of the detection of $\mathrm{H}_{2} \mathrm{O}_{2}$ with PEDOT:PSS/Pt NPs

OECT. Drain current recorded for $\mathrm{V}_{\mathrm{DS}}=-0.2 \mathrm{~V}$, upon addition of $\mathrm{H}_{2} \mathrm{O}_{2}$ when $\mathbf{b}$ ) the gate of the OECT is disconnected and c) with the gate polarized at $+0.4 \mathrm{~V}(\mathbf{d})$ gate current also reported). e) Evolution of the drain current versus time upon increasing the concentration of $\mathrm{H}_{2} \mathrm{O}_{2}$. Inset shows a zoom for low concentration in $\mathrm{H}_{2} \mathrm{O}_{2}$. f) Normalized current response of the OECT in function of the concentration of $\mathrm{H}_{2} \mathrm{O}_{2}$.

In order to elucidate the relative effects of $\mathrm{H}_{2} \mathrm{O}_{2}$ redox at the channel and gate, we first polarised only the channel $\left(\mathrm{V}_{\mathrm{DS}}=-0.2 \mathrm{~V}\right)$ with the gate disconnected. The corresponding current response is presented Figure 3b. After stabilisation of the drain current, $\mathrm{H}_{2} \mathrm{O}_{2}$ is added to the electrolyte $(0.91 \mathrm{mM})$, leading to a decrease of the drain current by $0.3 \mu \mathrm{A}$. Using a 


\section{WILEY-VCH}

separate OECT device, the channel was again polarised at $\mathrm{V}_{\mathrm{DS}}=-0.2 \mathrm{~V}$, but this time with the gate polarised at $+0.4 \mathrm{~V}$. The current response is shown Figure 3c. The same amount of $\mathrm{H}_{2} \mathrm{O}_{2}$ is added to the electrolyte after that the signal is stabilised. The addition of $\mathrm{H}_{2} \mathrm{O}_{2}$ generates a decrease in the drain current of $3.5 \mu \mathrm{A}$, which is a 10-fold higher signal than when only the channel is polarised. Although both phenomena $-\mathrm{H}_{2} \mathrm{O}_{2}$ reduction at the channel and oxidation at the gate - occur at the same time, the main component of the signal appears to arise from the oxidation of $\mathrm{H}_{2} \mathrm{O}_{2}$ at the PEDOT:PSS/Pt NPs gate, resulting in an stronger reduction of the channel. The gate current was recorded during the experiment and is presented Figure 3d. The addition of $\mathrm{H}_{2} \mathrm{O}_{2}$ increases the gate current from $10 \mathrm{nA}$ to $150 \mathrm{nA}$. A small modification in the gate current $(150 \mathrm{nA})$ generates a 20-fold larger modulation in the channel current $(3.5 \mu \mathrm{A})$.

The response of the OECT's drain current to successive addition of $\mathrm{H}_{2} \mathrm{O}_{2}$ is shown Figure 3e. The concentration of $\mathrm{H}_{2} \mathrm{O}_{2}$ in the electrolyte is gradually increased from $50 \mathrm{nM}$ to $500 \mu \mathrm{M}$. Additions are made every five minutes after a stable baseline signal (before adding any $\mathrm{H}_{2} \mathrm{O}_{2}$ ) is reached. There is a clear modification of the drain current when the concentration in $\mathrm{H}_{2} \mathrm{O}_{2}$ reaches $0.6 \mu \mathrm{M}$ (inset Figure 3e). Prior to this, the current is slowly decreasing as a consequence of the degradation of the OECT upon applying a continuous bias. In order to compare current response from different devices, one typically uses the normalized current response $\Delta \mathrm{I} / \mathrm{I}_{0}$ (Figure 3f). For each concentration, the value of the current was taken 2 min after adding the $\mathrm{H}_{2} \mathrm{O}_{2}$ solution. As seen in Figure 3f, the OECT devices show a limit of detection of $\mathrm{H}_{2} \mathrm{O}_{2}$ in the low $\mu \mathrm{M}$ range.

With sensitive detection of $\mathrm{H}_{2} \mathrm{O}_{2}$ established, we proceeded to experiments involving detection of the two neurotransmitters glutamate and acethylcholine. The amperometric detection of these substances by means of an oxydoreductase enzyme is typically achieved according to the mechanism depicted Figure 4a and Figure 4b. Glutamate is oxidised to 2- 


\section{WILEY-VCH}

oxoglutarate, reducing the GluOx, which is then reoxidised by oxygen. Oxygen is reduced in $\mathrm{H}_{2} \mathrm{O}_{2}$, which is then electrochemically oxidised at the electrode. The amount of $\mathrm{H}_{2} \mathrm{O}_{2}$ is directly proportional to that of the neurotransmitter. Because acethylcholine does not have a specific oxydoreductase, one typically needs to use a combination of two enzymes to achieve amperometric detection, making the system slightly more complex than in the case of glutamate. The first enzyme, AchE, transforms the acetylcholine into choline and acetate.

Choline is then oxidised by the Chox to betaine aldehyde and the enzyme is reoxidised by oxygen leading to the production of $\mathrm{H}_{2} \mathrm{O}_{2}$.
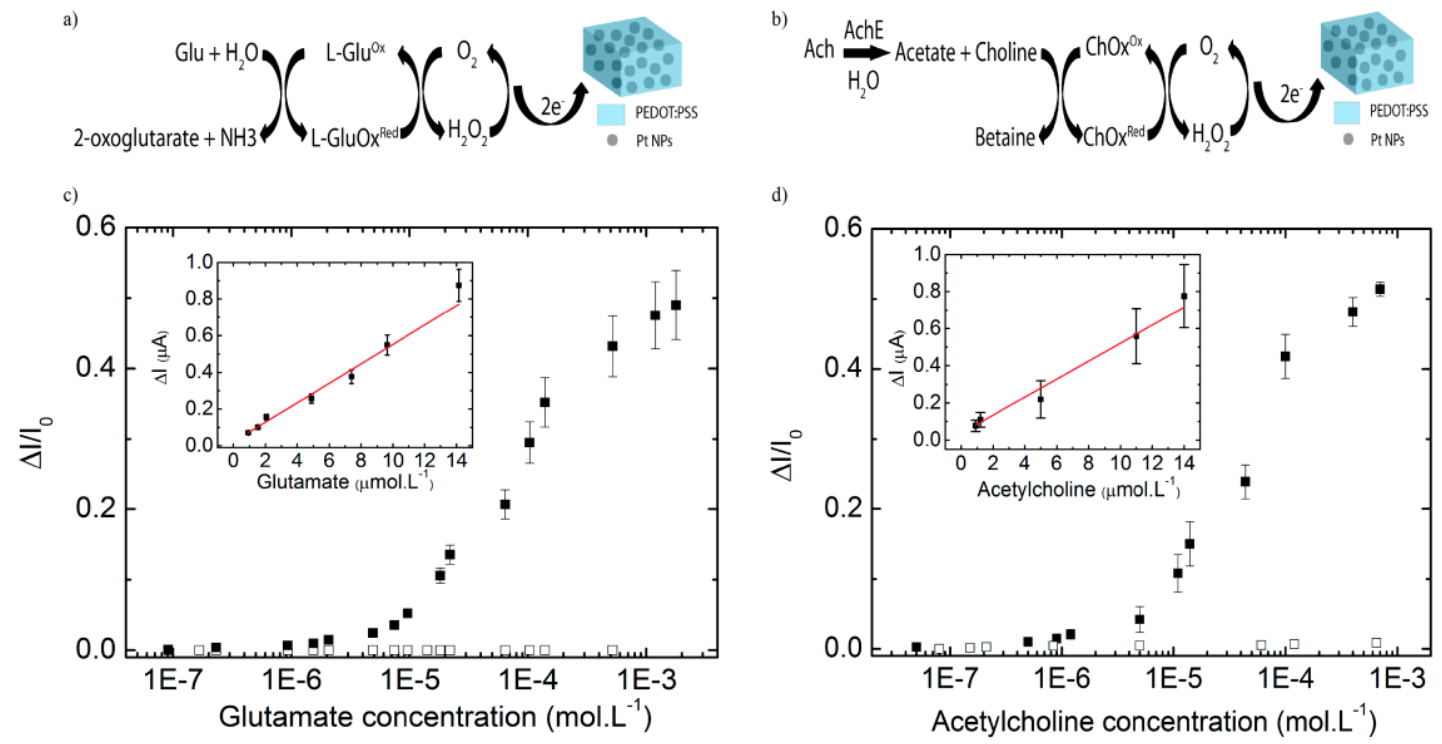

Figure 4. Reactions involved in the enzymatic detection of a) glutamate and b) acetylcholine.

Detection of c) glutamate (black squares) and d) acetylcholine (black squares) using PEDOT:PSS/Pt NPs OECT. Reference (empty squares) is measured by adding the analyte of interest on an OECT without any enzyme. The linear representations (insets $4 \mathrm{c}$ and $4 \mathrm{~d}$ ) were used to determine the sensitivity of the device.

The normalized current response curves are shown Figures $\mathbf{4 c}$ and $\mathbf{4 d}$ for glutamate and acethylcholine, respectively. As a reference, the response of an OECT (empty squares on Figures $\mathbf{4 c}$ and $\mathbf{4 d}$ ) without any enzyme immobilized was recorded as substrates were added. The reference curve allows us to monitor the effect of the various additions and stirring of the 


\section{WILEY-VCH}

solution and also to possible degradation of the drain current with a continuously applied voltage bias. For glutamate sensing, we found a sensitivity of $4.3 \mathrm{~A} \cdot \mathrm{mol}^{-1} \cdot \mathrm{L}^{1} \cdot \mathrm{cm}^{-2}$ (substrate concentration range from $0.9-14 \mu \mathrm{M}$ ). For ACh detection, a sensitivity of $4.1 \mathrm{~A} \cdot \mathrm{mol}^{-1} \cdot \mathrm{L}^{1} . \mathrm{cm}^{-2}$ can be estimated (substrate concentration range from $0.9-14 \mu \mathrm{M}$ ). The limits of detection were estimated using standard deviations from an averaged response curve. Accordingly, we found a limit of detection of $5 \mu \mathrm{M}$ for both glutamate and Ach. The detection limit of our OECT sensors is suitable for the detection of glutamate concentrations in the extracellular fluid, which are typically found in the low $\mu \mathrm{M}$ range. ${ }^{[33]}$ Although the detection limit is not sufficient for the detection of acetylcholine in the extracellular fluid, which is typically found in the $\mathrm{nM}$ range, ${ }^{[34]}$ our demonstration shows that the PEDOT:PSS/Pt NP OECTs can be used as sensors including a combination of two enzymes. Recent works ${ }^{[35]}$ have shown that it is possible to modify the shell of the NPs in order to make them more soluble in water. Current work, in our laboratory is performed to synthesise NPs in order to obtain a better dispersion of the nanoparticles in the PEDOT:PSS transducer matrix, hopefully leading to a lower detectable signal. Indeed, it is well documented that a good dispersion of the platinum nanoparticles within a matrix leads to a better catalytic activity of the nanoparticles towards the oxidation of $\mathrm{H}_{2} \mathrm{O}_{2} \cdot{ }^{[11]}$ This could potentially reduce the limit of detection of our sensors. The performance of our sensors could also be improved by optimizing the geometry of the OECT, in particular by using a gate area smaller than the channel area. ${ }^{[36]}$

\section{Conclusion}

In this work, we demonstrate that incorporation of platinum nanoparticles into PEDOT:PSS electrodes provides a simple method to manufacture OECTs for the selective and sensitive detection of neurotransmitters. Glutamate, the primary excitatory neurotransmitter in the central nervous system (CNS), could be detected down to concentrations found in the extracellular fluid. Acetylcholine, which forms the basis of the cholinergic regulatory system 


\section{WILEY-VCH}

in the CNS and is the primary activator of smooth muscle cells, could also be detected at low concentrations, however not low enough to enable monitoring of the neurotransmitter in the extracellular matrix in vivo. On-going work regarding the optimization of the dispersion of the nanoparticles in the PEDOT:PSS matrix is expected to improve the sensitivity considerably by increasing the available catalytic surface. Together, these results show that the OECT NP sensor technology can be made general for a variety of common oxydoreductase -based enzymatic sensing mechanisms, and could provide a route for future high-performance integrated sensing platforms.

\section{Experimental Section}

Materials: Choline oxidase (ChOx, EC 1.1.3.17), acetylcholine esterase (AchE, EC 3.1.1.7), L-glutamate oxidase (L-GluOx, EC 1.4.3.11), L-glutamic acid monosodium salt (98 \%), acetylcholine chloride (99\%), platinum (IV) chloride (99.99\%), hexylamine, sodium borohydride (99\%), 4,4'-diaminodiphenyl disulfide (98\%), 3-glycidoxypropyl trimethoxysilane (GOPS), dimethylsulfoxide, bovine serum albumine (BSA), phosphate buffered saline solution (PBS, ph=7.4) and glutaraldehyde (50\% wt in water) were purchased from Sigma-Aldrich and used without further purification. PEDOT:PSS (P Jet N V2) was purchased from Clevios.

Synthesis of the platinum nanoparticles: The platinum nanoparticles were synthesized according to the protocol developed by Perez et al. ${ }^{[37]}$ Platinum (IV) chloride (300 mg) was dissolved in hexylamine (75 mL). 4,4'-diaminodiphenyl disulfide (330 mg) was dissolved in a methanol/hexylamine mixture $(30 \mathrm{~mL}, 1 / 1)$. Finally, sodium borohydride (300 mg) was dissolved in a methanol/water mixture $(40 \mathrm{~mL}, 1 / 1)$ until complete dissolution of the sodium borohydride then hexylamine $(20 \mathrm{~mL})$ was added. The sodium borohydride solution was added to the platinum salt solution under stirring. $30 \mathrm{~s}$ after the solution turned brown, the disulfide solution was added and after $3.5 \mathrm{~min}$, DI water was added (200 mL). The solution 


\section{WILEY-VCH}

was left to stir for $15 \mathrm{~min}$. The organic phase was separated from the aqueous phase then washed repeatedly with DI water. The volume of the organic phase was then reduced by rotary evaporation down to $4 \mathrm{~mL}$. The solution was then poured into a centrifuge tube and 4,4'-diaminodiphenyl disulfide (300 mg) was added with ethanol (15 mL). The solution was left to stir overnight. The nanoparticles were recovered by centrifugation in diethyl ether. Preparation of the PEDOT:PSS/Pt NPs composite films: The composite films of PEDOT:PSS/Pt NPs were prepared by spin-casting a solution of PEDOT:PSS to which was added $5 \%$ wt of the solution of Pt NPs dispersed in DMSO $\left(5 \mathrm{mg}^{\mathrm{mL}} \mathrm{mL}^{-1}\right)$ and $0.2 \%$ wt of GOPS, to prevent delamination of the composite films when immersed in the electrolyte solution.

TEM measurements: A droplet of the Pt NPs in DMSO solution was put on a carbon-coated copper TEM grid and left to evaporate. The composite films were formed on a glass slide coated with PMMA, which was used as a sacrificial layer. PMMA was dissolved in acetone, leaving the PEDOT:PSS/Pt NPs film to float. The film was put on a copper grid for TEM measurements, which were performed on a FEI Tecnai G2.

Electrochemical characterization: Cyclic voltammetry and chronoamperometry were performed using a microAutolab potentiostat. A three-electrode configuration was used. The reference electrode used was a commercial silver/silver chloride $(\mathrm{Ag} / \mathrm{AgCl})$ electrode and the counter electrode was a platinum mesh. The polymer films were formed on an ITO electrode. Nail polish was painted on the electrode to define a 1-cm2 surface in contact with the electrolyte. PBS was used as electrolyte.

Manufacturing of OECTs: A 4 in glass wafer was thoroughly cleaned by sonication in DI water with detergent, acetone and isopropyl alcohol. The substrate was then dried and underwent a UV-ozone treatment. The PEDOT:PSS/Pt NPs blend spin-cast on the substrate and annealed at $140{ }^{\circ} \mathrm{C}$ for $1 \mathrm{~h}$. Then the substrate was put in DI water for $1 \mathrm{~h}$ to remove the short, soluble PEDOT:PSS chains. The channel and the source, drain and gate electrodes were 


\section{WILEY-VCH}

patterned with S1805 photoresist, exposed to UV light using a Karl Süss MA6/BA6 mask aligner, and developed using MF-319 developer. The OECT pattern was obtained by dry etching, with oxygen plasma, the PEDOT:PSS not protected by the S1805. The photoresist was subsequently removed in acetone. The electrolyte area and the contact pads were defined using SU-8 photoresist and mr-Dev 600 as developer. Finally, carbon paste was painted on the contact pads for the gate, source and drain in order to have a better electrical contact between the probes and the electrodes. The channel length and width were $2500 \mu \mathrm{m}$ and $500 \mu \mathrm{m}$, respectively, giving a surface area of $0.0125 \mathrm{~cm}^{2}$. The gate area was $0.075 \mathrm{~cm}^{2}$. Immobilization of the enzymes: GluOx was immobilized on the gate of the OECT by crosslinking with BSA and glutaraldehyde. The immobilization solution was prepared by adding $20 \mu \mathrm{L}$ of a solution of glutamate oxidase at $0.1 \mathrm{U} \cdot \mathrm{mL}^{-1}$ to $100 \mu \mathrm{L}$ of PBS. $2.5 \mathrm{mg}$ of BSA and $10 \mu \mathrm{L}$ of glutaraldehyde solution (2.5\% wt) were added to this solution. $3 \mu \mathrm{L}$ of this solution were placed on the gate of the OECT. The solution was left to dry at room temperature for $24 \mathrm{~h}$ then thoroughly washed with PBS to remove any non-immobilized enzyme. Devices were then stored in PBS solution at $+6{ }^{\circ} \mathrm{C}$ for $24 \mathrm{~h}$ before testing. For ACh detection, a similar solution was prepared by using a mixture of $\mathrm{AChE}$ and $\mathrm{ChOx}$ instead of GluOx.

Device characterization: All electrical characterizations were done using PBS as electrolyte. Output and transfer characteristics were obtained using a Keithley K4200. The current versus time curves were recorded using a Keithley K2612A with custom LabVIEW software.

\section{Acknowledgements}

L. K. thanks the the EU Seventh Framework Programme Marie Curie (PIEF-GA-2011301796) Project OEAN for funding. M. B. wishes to thank the Önnesjö foundation for funding. We would also like to thank the Swedish Research Council (grant 2002-4497), the Swedish Foundation for Strategic Research (grant IMF11-0052) and the Knut and Alice 
Wallenberg Foundation (grant Wallenberg Scholar 2012-0302) for funding. B. P., M. C. P. and V. N. thank Campus France (PHC Dalen 2011-\#26218VC).

We thank Jun Lu for the TEM measurements.

Received: ((will be filled in by the editorial staff))

Revised: ((will be filled in by the editorial staff)) Published online: ((will be filled in by the editorial staff))

[1] World Health Organization, Neurological Disorders: Public health challenges. 2006.

[2] Q. R. Smith, J. Nutr. 2000, 130, 1016.

[3] P. Kása, Z. Rakonczay, K. Gulya, Prog. Neurobiol. 1997, 52, 511.

[4] R. M. Marchbanks, Psychol. M. 1980, 10, 1.

[5] L. Bianchi, L. D. Corte, and K.F. Tipton, J. Chromatogr. B. 1999, 723, 47.

[6] M. G. Giovannini, D. Mutolo, L. Bianchi, A. Michelassi, G. Pepeu, J. Neurosci.1994, 14, 1358.

[7] S. B. Hall, E. A. Khudaish, A. L. Hart, Electrochim. Acta 1997, 43, 579.

[8] K. Hascup, E. C. Rutherford, J. E. Quintero, B. K. Day, J. R. Nickell, F. Pomerleau, P. Huettl, J. J. Burmeister, G. A. Gerhardt, in Electrochemical methods for Neuroscience (Eds: A. C. Michael, L. M. Borland), CRC Press, Boca Raton, FL, USA 2007, Ch. 19.

[9] M. E. Sandison, N. Anicet, A. Glidle, J. M. Cooper, Anal. Chem. 2002, 74, 5717.

[10] T. You, O. Niwa, M. Tomita, S. Hirono, Anal. Chem. 2003, 75, 2080.

[11] L. Challier, F. Gal, G. Carot, H. Perez, V. Noël, Electrochem. Commun. 2013, 28, 118.

[12] C. C. Chang, M. T. Jiang, C. L. Chang, C. L. Lin, Mater. Chem. Phys. 2011, 127, 440.

[13] D. Svirskis, J. Travas-Sejdic, A. Rogers, S. Garg, J. Control. Release 2010, 146, 6.

[14] D.T. Simon, S. Kurup, K. C. Larsson, R. Hori, K. Tybrandt, M. Goiny, E. W. H. Jager, M. Berggren, B. Canlon, A. Richter-Dahlfors, Nat. Mater. 2009, 8,742.

[15] H. S. White, G. P. Kittlesen, M. S. Wrighton, J. Am. Chem. Soc. 1984, 106, 5375.

[16] S. R. Forrest, Nature 2004, 428, 911.

[17] K. Tybrandt, K. C. Larsson, S. Kurup, D. T. Simon, P. Kjäll, J. Isaksson, M. Sandberg, E. W. H. Jager, A. Richter-Dahlfors, M. Berggren, Adv. Mater. 2009, $21,4442$.

[18] D. Tobjörk, R. Österbacka, Adv. Mater. 2011, 23,1935.

[19] M. Hamedi, R. Forchheimer, O. Inganäs, Nat. Mater. 2007, 6, 357.

[20] J. Isaksson, P. Kjäll, D. Nilsson, N. Robinson, M. Berggren, A. Richter-Dahlfors, Nat. Mater. 2007, 6, 673.

[21] M. H. Bolin, K. Svennersten, D. Nilsson, A. Sawatdee, E. W. H. Jager, A. RichterDahlfors, M. Berggren, Adv. Mater. 2009, 21, 4379.

[22] L. H. Jimison, S. A. Tria, D. Khodagholy, M. Gurfinkel, E. Lanzarini, A. Hama, G. G. Malliaras, R. M. Owens, Adv. Mater. 2012, 24, 5919.

[23] D. Khodagholy, T. Doublet, P. Quilichini, M. Gurfinkel, P. Leleux, A. Ghestem, E. Ismailova, T. Hervé, S. Sanaur, C. Bernard, G. G. Malliaras, Nat. Commun. 2013, 4, 1575.

[24] P. Lin, X. Luo, I. M. Hsing, F. Yan, Adv. Mater. 2011, 23, 4035.

[25] H. Tang, P. Lin, H. L. W. Chan, F. Yan, Biosens. Bioelectron. 2011, 26, 4559.

[26] R. X., He, M. Zhang, F. Tan, P. H. M. Leung, X. Z. Zhao, H. L. W. Chan, M. Yang, F. Yan, J. Mater. Chem. 2012, 22072. 


\section{WILEY-VCH}

[27] S. Y. Yang, J. A. DeFranco, Y. A. Sylvester, T. J. Gobert, D. J. Macaya, R. M. Owens, G. G. Malliaras, Lab on a Chip 2009, 9, 704.

[28] D.A. Bernards, G. G. Malliaras, Adv. Func. Mater. 2007, 17, 3538.

[29] H. Tang, F. Yan, P. Lin, J. Xu, H. L. W. Chan, Adv. Mater. 2011, 21, 2264.

[30] M. C. Pham, B. Piro in Iontronics, Ionic Carriers in Organic Electronic Materials and Devices (Eds: J. Leger, M. Berggren, S. Carter), CRC Press, Boca Raton, FL, USA 2010, Ch.4.

[31] S. D. Uzun, Fatma Kayaci, T. Uyar, S. Timur, L. Toppare, ACS Appl. Mater. Inter. 2014, 6, 5235 .

[32] G. Yang, K. L. Kampstra, M. R. Abidian, Adv. Mater. 2014, DOI: 10.1002/adma.201400753

[33] N. C. Danbolt, Prog. Neurobiol. 2001, 65,1.

[34] P. Uutela, R. Reinilä, R. A. Ketola, R. Kostiainen, Rapid Commun. Mass Sp. 2005, 19, 2950.

[35] F. Gal, H. Perez, V. Noël, G. Carrot, J. Polym. Sci. Pol. Chem. 2012, 50, 289.

[36] F. Cicoira, M. Sessolo, O. Yaghmazadeh, J. A. DeFranco, S. Y. Yang, G. G. Malliaras, Adv. Mater. 2010, 22, 1012.

[37] H. Perez, J. P. Pradeau, P. A. Albouy, J. Perez-Omil, Chem. Mater. 1999, 11, 3460. 